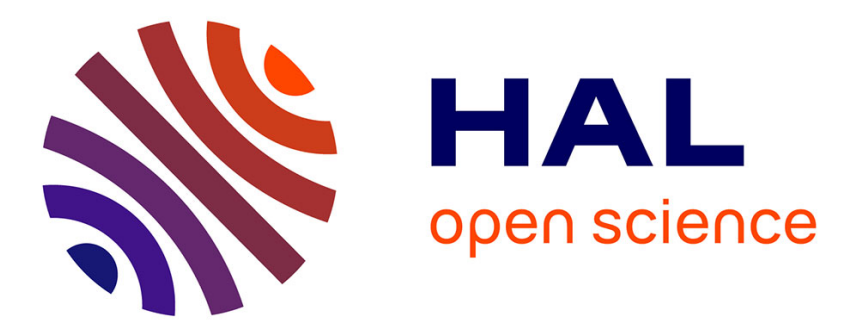

\title{
Storage of Carotenoids in Crustaceans as an Adaptation to Modulate Immunopathology and Optimize Immunological and Life-History Strategies
}

Aurélie Babin, Jérôme Moreau, Yannick Moret

\section{- To cite this version:}

Aurélie Babin, Jérôme Moreau, Yannick Moret. Storage of Carotenoids in Crustaceans as an Adaptation to Modulate Immunopathology and Optimize Immunological and Life-History Strategies. BioEssays, 2019, 41 (11), pp.1800254. 10.1002/bies.201800254 hal-02369709

\author{
HAL Id: hal-02369709 \\ https://hal.science/hal-02369709
}

Submitted on 27 Nov 2020

HAL is a multi-disciplinary open access archive for the deposit and dissemination of scientific research documents, whether they are published or not. The documents may come from teaching and research institutions in France or abroad, or from public or private research centers.
L'archive ouverte pluridisciplinaire HAL, est destinée au dépôt et à la diffusion de documents scientifiques de niveau recherche, publiés ou non, émanant des établissements d'enseignement et de recherche français ou étrangers, des laboratoires publics ou privés. 
Storage of carotenoids in crustaceans as an adaptation to modulate immunopathology and optimize immunological and life-history strategies

Aurélie Babin*, Jérôme Moreau, Yannick Moret*

Équipe Écologie Évolutive, UMR CNRS 6282 Biogéosciences, Université Bourgogne Franche-Comté, 6 Boulevard Gabriel, F-21000 Dijon, France

*Corresponding authors: aurelie.babin@hotmail.fr ; yannick.moret@u-bourgogne.fr

Manuscript type: Insights and Perspectives / Hypothesis manuscript

Running title: Carotenoids and evolutionary strategies

Keywords: carotenoids, immune cost, resistance, tolerance, ageing, invertebrate

Word counts: Abstract: 148; Main text: 5 123; Figure legends: 429

References: 128

Figures: 3 
Why do some invertebrates store so much carotenoids in their tissues? Storage of carotenoids may not simply be passive and dependent on their environmental availability, as storage variation exists at various taxonomic scales, including among individuals within species. While the strong antioxidant and sometimes immune-stimulating properties of carotenoids might be beneficial enough to cause the evolution of features improving their assimilation and storage, they may also have fitness downsides explaining why massive carotenoid storage is not universal. Here, we examine the functional and ecological implications of carotenoid storage for the evolution of invertebrate innate immune defences, especially in crustaceans, which massively store carotenoids for unclear reasons. We propose three testable hypotheses about the role of carotenoid storage in immunological (resistance and tolerance) and lifehistory strategies (with a focus on ageing), which may ultimately explain the storage of large amounts of these pigments in a context of host-pathogen interactions. 


\section{Introduction}

Carotenoids are natural lipid-soluble bioactive pigments, produced by photosynthetic organisms (plants, bacteria, and algae) in which they absorb light for photosynthesis and provide photoprotection. ${ }^{[1]}$ Apart from the rare exceptions involving symbiotic interactions with microorganisms, ${ }^{[2,3]}$ animals cannot produce carotenoids themselves and must acquire them from their diet. ${ }^{[1,4]}$ There are over 600 carotenoids known as xanthophylls and carotenes when containing or not oxygen, respectively. ${ }^{[4]}$ Despite such a great diversity of carotenoids in nature, only a few of them are absorbed and stored in animal tissues and fluids. ${ }^{[5-7]}$ This suggests that they are either selectively accumulated by animals and/or metabolically transformed for their storage. ${ }^{[8]}$ The amounts of carotenoids stored by animals vary across $\operatorname{taxa}^{[9,10]}$ and within species, ${ }^{[11]}$ suggesting variability in their physiological importance in addition to fluctuations in their environmental availability.

Because they are involved in many biological functions, ${ }^{[4,5]}$ carotenoids may have strong implications in evolutionary biology. Their storage was suggested as indicative of individual quality, such that carotenoid-colored traits could be sexually selected. ${ }^{[12]}$ They have been found to have beneficial effects on survival, growth, and immunity, although this is still strongly debated, especially in the bird literature from which most of the studies were conducted..$^{[5,13-17]}$ Non oxygenated carotenoids, such as beta-carotene can be important precursors to vitamin A in many animals, whereas oxygenated ones, also called xanthophyll carotenoids, such as astaxanthin and lutein, are not. ${ }^{[18-20]}$ Astaxanthin, mainly produced by fungi and algae, is massively stored by aquatic animals. ${ }^{[8]}$ Specifically, most crustaceans accumulate massive amounts of astaxanthin as circulating lipid droplets in the haemolymph and in the eggs, and as esterified forms in their tissues. ${ }^{[7,21-23]}$ This pigment, rarely found in large amounts in vertebrates except for reports in salmonid fish ${ }^{[8,24-26]}$, is essentially produced from the metabolism of other carotenoids acquired in the diet ${ }^{[27,28]}$ and is among the 
carotenoids having the highest antioxidant potential. ${ }^{[29]}$ The precise reasons for such substantial pigment accumulation in crustaceans remain unclear. However, its accumulation has not been related so far to sexual signalling. Furthermore, while the involvement of carotenoids as antioxidants in birds appears inconclusive (Koch et al. 2018), astaxanthin in crustaceans, by contrast, may act as an important antioxidant (Ambati et al., 2014; Snoeijs and Häubner, 2014; Schneider et al., 2016; de Carvalho and Caramujo, 2017; Weaver et al. 2018). We propose that the strong antioxidant and the frequent immune-stimulating roles of astaxanthin may limit the immunopathology cost associated with the immune response of crustaceans. $^{[11,30,31]}$

Parasitism is an important source of stress for organisms. While the immune defences protect from parasitic and pathogenic agents (here after pathogens), inflammatory responses from the innate immune system also cause immunopathological disorders. Inflammation is a phenomenon known from both vertebrates and invertebrates, corresponding to a fast but nonspecific response characterised by the delivery of fluids, cytotoxic chemicals and cells to damaged and infected tissues, in order to fend off infectious agents and initiate tissue repair. The cytotoxic chemicals released by the respiratory burst comprise highly reactive oxygen species (ROS) and reactive nitrogen species (RNS) toxic to both pathogens and hosts' tissues, leading to immunopathology. ${ }^{[32]}$ When damaged tissues are not fully repaired and homeostasis is not fully restored, inflammation can further develop into a chronic condition, with inevitable long-term debilitating consequences, such as increased rates of morbidity and mortality at older age. ${ }^{[32]}$

Organisms have evolved physiological processes that reduce the impact of inflammatory injury, thus limiting the immunopathology costs. The integrated antioxidant defence system controls the balance between the production and the detoxification of cytotoxic free radicals, produced by basal metabolic activity and the immune response. This 
system combines endogenous antioxidant enzymes, such as superoxide dismutase, catalase and glutathione peroxidase, with exogenous (diet-acquired) antioxidants, such as vitamin $\mathrm{E}$ (tocopherol), vitamin C (ascorbate), polyphenolic antioxidants, and carotenoids. ${ }^{[33,34]}$ Among these compounds, carotenoids may scavenge free radicals produced by immune activity because of their long carbon skeleton containing conjugated double bonds and, in some cases, oxygenated end rings. ${ }^{[35-38]}$ They also have the potential to interact with endogenous antioxidant enzymes. $^{[31,39,40]}$

Here, we emphasize that the environmental availability of carotenoids and the ability of organisms to assimilate and store these pigments, may impact the evolution of innate immunity, by improving the defences against pathogens while reducing the fitness costs associated with the inflammation response. We particularly focus on crustaceans for several reasons, developed in the dedicated section below: their immunity relies on innate immune systems alone, thus leading to immunopathology ${ }^{[41]}$; they are known to accumulate naturally large amounts of astaxanthin, a carotenoid with a particular high antioxidant ability ${ }^{[29]}$,which is also frequently reported to enhance their immunity. ${ }^{[31,42-45]}$; and carotenoid storage in crustaceans has not been involved so far in communication displays, which simplifies the investigation of its functions. We first briefly review the mechanisms underlying the invertebrate inflammatory response, with a special focus on the production of ROS and RNS. After describing the integrated antioxidant defence system that includes dietary carotenoids, we discuss the role of astaxanthin in modulating immunopathology, immunological strategies (tolerance versus resistance) and ageing. Finally, we explore whether crustaceans could have evolved the ability to store large amounts of astaxanthin in their tissues to maintain higher levels of innate immune defences at lower immunopathological costs in response to local pathogens. 


\section{Innate immunity and immunopathology in invertebrates}

Invertebrate innate immunity is a costly system of protection that detects and eliminates a wide variety of pathogens and unhealthy tissues. It comprises constitutive defences relying on immune cells (haemocytes or coelomocytes) and several rapidly inducible enzymatic cascades (e.g. the prophenoloxidase cascade) at the core of the inflammatory response. ${ }^{[46]}$ Upon infection, recruited phagocytic immune cells increase their oxygen consumption during the respiratory burst that leads to ROS production (Fig. 1), enhancing pathogen killing within phagocytic cells and in the vicinity of extracellular bound pathogens. ${ }^{[47]}$ ROS are produced by the activity of the membrane bound NADPH oxidase enzymatic complex, which catalyses the production of $\mathrm{NADP}^{+}$and cytotoxic superoxide anions $\left(\mathrm{O}_{2}{ }^{-}\right)$. Superoxide anions can then be transformed into other damaging and unstable molecules, such as hydroxyl radicals $\mathrm{OH}^{-}$, and hydrogen peroxide $\mathrm{H}_{2} \mathrm{O}_{2}$ forming hypochlorous acid $\mathrm{HOCl}$ in reaction with chloride. ${ }^{[48]}$ Recruited immune cells can also generate RNS, such as nitric oxide (NO). ${ }^{[49,50]}$ In immune cells, the synthesis of NO by inducible nitric oxide synthase, iNOS, has a broad antipathogenic action (Fig. 1). NO can react with ROS yielding other highly reactive radicals (Fig. 1): combined with hydrogen peroxide, it yields hydroxyl radicals. $;^{[48,51]}$ combined with superoxide anions, it yields oxidant and nitrating peroxynitrite radicals, ONOO-, that can subsequently damage a large range of the cell molecules, inducing apoptotic or necrotic cell death. ${ }^{[48,52]}$

A major response of invertebrate immunity accompanying the rapid aggregation of the immune cells with each other and with the pathogens is the melanisation reaction catalysed by phenoloxidase enzymes (PO), which heal wounds through clotting and clear invading pathogens through nodulation or encapsulation. ${ }^{[53]}$ POs are present as inactive proenzymes (prophenoloxidase or proPO) in immune cells. They are released and converted into their active form in the haemolymph upon wounding or infection. In crustaceans, infection may 
further generate the cleavage of a fragment of the hemocyanin, a respiratory protein, which also has PO activity. ${ }^{[54]}$ Melanin synthesis is accompanied by the production of ROS and RNS, helping to kill intruders. ${ }^{[50,53]}$ However, excessive PO cascade activity can subsequently damage the host's own tissues and organs (Fig. 2). ${ }^{[55,56]}$ Immunopathological effects upon encapsulation and clotting are likely to be frequent in invertebrates since the occurrence and abundance of wounding can be extremely high. ${ }^{[57-59]}$ Repeated wound clotting and multiple melanisation reactions in response to repeated infections may be then harmful, as it exposes the host organs to cumulative cytotoxic radicals in the open circulating system.

\section{Carotenoids as part of the integrated antioxidant defence system}

The accumulation of cytotoxic ROS and RNS is limited by the detoxifying activity of the integrated antioxidant system, including endogenous enzymes and non-enzymatic exogenous compounds. ${ }^{[33,60]}$ Three major antioxidant enzymes detoxify free radicals (see Fig. 1).

Superoxide dismutase transforms highly reactive superoxide anions $\mathrm{O}_{2}{ }^{--}$into hydrogen peroxide. Then catalase converts hydrogen peroxide into water. Hydrogen peroxide detoxification can also be catalysed by peroxidase when ROS are associated with substrates that can be oxidised. ${ }^{[33,61]}$ In addition to these three major enzymes, crustaceans possess peroxinectins, proteins with multiple roles in immune defences, including peroxidase activity. Upon immune challenge, peroxinectins are released by haemocytes after the activation of the proPO cascade, bound to extracellular superoxide dismutase, thus helping to detoxify the hydrogen peroxide produced by superoxide dismutase activity. ${ }^{[62,63]}$ However, peroxinectins seem to have lower peroxidase activity than catalase upon immune challenge. ${ }^{[31]}$ Non-enzymatic exogenous compounds with antioxidant properties are acquired through dietary intake and presumably help antioxidant enzymes to keep the oxidative burden under control. These compounds act either as hydrogen donors (chemical traps), or as free 
radical scavengers (physical traps) ${ }^{[33,60,64]}$ Non-enzymatic antioxidants are divided into hydrophilic compounds, present in aqueous cellular compartments (e.g. vitamin C, glutathione), and lipophilic compounds (e.g. carotenoids), which can be included in cell membranes (Fig. 1) and associated with lipoproteins. ${ }^{[33,60]}$ All these exogenous antioxidant compounds being acquired from the diet, their environmental availability can be limited and limiting. Hence, this environmental availability, as well as the ability to assimilate these antioxidant compounds, will be a crucial parameter influencing the potential synergy with endogenous antioxidant enzymes and in the general antioxidant capacity. Among these compounds, carotenoids are powerful antioxidants ${ }^{[35,36]}$ and relatively abundant in natural food sources. ${ }^{[65]}$ Unlike other dietary antioxidants, they can be stored in large amounts in animal tissues. ${ }^{[7,66]}$ Hence, carotenoids potentially play a major role in the regulation of immunopathology, and therefore in the modulation and the evolution of immune defences.

\section{Crustaceans as systems for studying physiological and evolutionary implications of}

\section{carotenoid storage}

Along with their biological proprieties, carotenoids are involved in several functions including in communication displays in birds, amphibians, and reptiles. Carotenoid-based coloration of the skin and plumage has been found to be an honest secondary signal of resistance to oxidative stress, body condition, and immunocompetence. ${ }^{[5,25]}$ Several hypotheses on the molecular mechanisms underlying signal honesty have been formulated, including the allocation trade-offs and shared molecular pathways, leading to specific expected relationships between stored amounts of carotenoids and other physiological parameters. ${ }^{[15,67,68]}$ Nevertheless, it has been suggested that stored carotenoids may be minor antioxidants for birds, preferentially used for sexual signalling, while other non-pigmentary antioxidants (e.g. melatonin) may be used primarily for detoxification during immune 
activity. ${ }^{[69]}$ In these bird examples, carotenoid-based coloration might have been under strong selection, leading to diversion of these pigments for sexual signalling. Therefore, taxa that do not use these pigments for communication displays would be more straightforward to study the role of carotenoids in alleviating oxidative stress in immunopathology, avoiding interferences with signalling.

In invertebrates, aquatic crustaceans are among the greater animal reservoirs of carotenoids by accumulating mostly astaxanthin in their tissues, cuticle, haemolymph, and eggs. ${ }^{[7,9,22,28]}$ They can obtain this pigment in their diet by feeding on various algae and microbes that are producing them ${ }^{[8-10]}$ However, the most common carotenoids contained in their food are typically beta-carotene, zeaxanthin, lutein, and other less abundant carotenoids that crustaceans are oxidizing to produce the astaxanthin they accumulate. ${ }^{[27,28]}$ They can also directly obtain astaxanthin by consuming animals that have already performed the bioconversion of other carotenoids into astaxanthin and stored it. Astaxanthin has the highest antioxidant activity among natural carotenoids: it is 10 times more effective than $\beta$-carotene and over 100 times more effective than vitamin $E \cdot{ }^{[70-72]}$ However, the presence of salt lowers the relative oxidant activity of astaxanthin. ${ }^{[73]}$ Assuming that animals store astaxanthin mainly for its antioxidant potential, it may not be surprising that some marine organisms (crustaceans, but also some fish, see the introduction) store them in large amounts to compensate for its reduced antioxidant activity in salty water, although this needs to be formally tested. Astaxanthin also has unique other biochemical properties making it able to form chelate complexes with metals that could otherwise impair mitochondrial function. ${ }^{[73]}$ Its ability to be esterified allows a better stabilization of the free form and prevents its aggregation that would otherwise favour pro-oxidant effects. ${ }^{[29]}$ The latter phenomenon could be further prevented by the ability of the pigment to form proton loss neutral radicals. ${ }^{[29]}$ Together, these properties likely enable the accumulation of large amounts of astaxanthin 
with limited negative effects, making it an efficient protective agent against environmental stressors.

The natural storage of large quantities of astaxanthin seems to be the healthy/normal situation for crustaceans, whereas the food depletion in carotenoids seems to be unusual; the astaxanthin storage rate depending on the pigment nature and availability. ${ }^{[28]}$ In freshwater and marine crustaceans, the storage of astaxanthin was associated to numerous beneficial physiological effects, including immunity ${ }^{[74-76]}$ and antioxidant defences ${ }^{[77,78]}$ but has never been reported to be involved to sexual signalling, either directly or indirectly. By contrast with vertebrate and insect immunity, crustacean immunity relies strongly on hemocytes, the cytotoxic activity of the proPO cascade and on the production of ROS/RNS. The large panel of antimicrobial peptides described in insects, allowing them to efficiently control microbial infections without generating self-damage, has no equivalent, so far, in crustaceans. ${ }^{[79]}$ Crustacean immunity is therefore strongly dependent on self-reactive processes to combat infection by pathogens. Furthermore, crustaceans have an open circulatory system frequently exposed to microbes often in high concentrations in their natural habitat. ${ }^{[80-82]}$ All these elements suggest that storage of carotenoids has a central role in the physiology of crustaceans, which make then an important model system to study the role of carotenoids in the detoxification of the immune response.

\section{Hypothesis 1: carotenoids modulate immunological strategies of crustaceans by limiting immunopathology}

Animals may survive infection through two non-mutually exclusive immunological strategies: resistance and tolerance. ${ }^{[83,84]}$ Resistance reduces pathogen burden by the action of the host's immune system, but leads to significant immunopathology, and hence to fitness costs (see above). Alternatively, the host may restrain its immune response and tolerate a certain 
pathogen burden, limiting the immunopathology associated with the immune response. These two immunological strategies could be closely linked, as limiting immunopathology (tolerance strategy) may allow the host's immune system to be more efficient at eliminating pathogens (resistance strategy). ${ }^{[85]}$ Therefore, any mechanism limiting immunopathology is expected to have profound implications for the evolution of tolerance and/or resistance, ${ }^{[32,86]}$ and for host-pathogen interactions. ${ }^{[85]}$

Here, we propose that the immune-stimulant and antioxidant activities of carotenoids could influence the crustacean's resistance and tolerance immunological strategies, by enhancing immune response efficiency while limiting immunopathology. This would translate into a very efficient and lower-cost immune function resulting from the storage of the storage of large amounts of astaxanthin. ${ }^{[30,31]}$ Carotenoids could improve resistance as they were reported to stimulate immune defences of vertebrate animals ${ }^{[\mathrm{e.g.} .5,15,16,87]}$, although this is not always the case. ${ }^{[14,37,38,88]}$ In marine and freshwater crustaceans, immunity is frequently improved by dietary carotenoids. ${ }^{[31,42-45]}$ For instance, in the amphipod crustacean Gammarus pulex, experimental dietary supplementation with carotenoids enhanced immune activity and increased resistance to microbial infection (Fig. 2). ${ }^{[30]}$ Dietary supplementation with carotenoids increases phenoloxidase activity and total haemocyte count in the giant freshwater prawn Macrobrachium rosenbergii. ${ }^{[89]}$ Furthermore, the injection of astaxanthin in the same species increases total haemocyte count and survival when exposed to the pathogenic bacterium Lactococcus garvieae. ${ }^{[90]}$ Likewise, a dietary supplementation with astaxanthin increased the total haemocyte count of the marine whiteleg shrimp Litopenaeus vannamei juveniles. ${ }^{[43]}$ Similarly, a yeast-rich diet containing carotenoids increased total haemocyte count and phenoloxidase activity in the marine Indian prawn Fenneropenaeus indicus. ${ }^{[4]}$ The mechanisms through which carotenoids directly or indirectly enhanced basal levels of immune effectors are still unknown. Nevertheless, recent findings suggest that 
astaxanthin exhibits antimicrobial activity against a large range of microbes in vitro through yet unknown mechanisms. ${ }^{[1,92]}$ Storage of this pigment could therefore provide a direct advantage upon microbial infection.

Carotenoids could also improve tolerance in several ways. They may prevent oxidative stress-mediated damage resulting from the immune response by scavenging cytotoxic free radicals (Fig. 1), ${ }^{[35]}$ helping in their detoxification, ${ }^{[36]}$ hence limiting the immunopathology cost (Fig. 3). This might be particularly important in crustaceans whose immunity strongly relies on auto-reactive immune responses in an open circulating system where the organs bathe in the haemolymph. These processes are suspected to occur in carotenoid-supplemented G. pulex, which do not suffer from additional survival costs compared to control gammarids, despite exhibiting stronger immune activity and resistance to microbes. ${ }^{[30]}$ From a functional perspective, improved tolerance mediated by carotenoids in G. pulex might result from the take-over of the activity of the antioxidant enzyme superoxide dismutase in the detoxification of the superoxide anions of the innate immune response, while the activity of the other antioxidant enzyme catalase is promoted. ${ }^{[31]}$ Nevertheless, further studies are needed to better understand the functional role of carotenoids in the detoxification process in association with endogenous antioxidant enzymes.

Carotenoid-mediated tolerance may also result from interactions between the pigments and the regulation of the host's metabolism. ${ }^{[19,93]}$ Infections can have dramatic metabolic consequences, characterised by the loss of energetic reserves. This results in elevated circulation of glucose, amino acids and fatty acids, which can cause morbidity and death. ${ }^{[94,95]}$ Carotenoids, especially astaxanthin, have hypolipidemic and hypoglycemic effects in rodents after supplementation. ${ }^{[19,96]}$ Moreover, there is evidence that astaxanthin can interact with nuclear receptors of the peroxisome proliferator-activated receptor (PPAR) superfamily, which regulates lipid and glucose metabolism. ${ }^{[97]}$ It is therefore possible that astaxanthin may 
prevent metabolism dysregulation upon infection through interaction with PPARs. If so, carotenoids may not only improve tolerance by preventing wasting of metabolic reserves, but also impact resource trade-offs involving the immune function upon immune challenge.

Beyond the functional characterisation of the interactions of carotenoids with the host physiology, testing the above hypothesis relies on determining the phenotypic consequences of the dietary supplementation with the pigments. Especially, under the above hypothesis, the supplementation of the food of crustaceans with carotenoids is expected to improve the survival and reproductive success of hosts exposed to infectious or non-infectious immune challenges if the pigments enhance immunity or tolerance, respectively.

\section{Hypothesis 2: carotenoids modulate ageing in crustaceans by limiting immunopathology}

Ageing is the progressive deterioration of physiological functions with age, leading to agedependent decline in fecundity and increased mortality. ${ }^{[98]}$ Several non-mutually exclusive hypotheses have been put forward to explain ageing, and many involve cumulative oxidative damage by biological processes. ${ }^{[99,100]}$ Mechanisms such as antagonistic pleiotropy, when the expression of genes is beneficial early in life but becomes adverse at older age, ${ }^{[101,102]}$ and damage by ROS and RNS, all contribute to ageing. ${ }^{[103,104]}$ Ageing and inflammation-mediated immunopathology are intrinsically linked, as ageing is characterised by a prolonged proinflammatory status as a result of an imbalance between pro- and anti-inflammatory status. As stated above, ROS and RNS accumulation is a prominent theory of ageing, ${ }^{[103,104]}$ and their regulation in the host when interacting with pathogens appears critical in the ageing process. ${ }^{[32,56]}$ It has been proposed that early-life inflammation could have long-term or delayed pathological costs, yielding increased morbidity and mortality at older age. ${ }^{[105,106]}$ In line with this, a non-pathogenic immune response experimentally elicited in early life in the mealworm beetle, T. molitor, reduces adult life span. ${ }^{[32]}$ In this insect, activation of the 
prophenoloxidase cascade and the release of ROS and RNS by haemocytes in response to non-self have an immunopathological effect on Malpighian tubules (insect 'kidneys'), leading to a lower secretion efficiency. Their function is impaired on the long term, ${ }^{[55]}$ causing ageing acceleration $^{[56]}$ and shortening lifetime reproductive success. Preventing immunopathology early in life may reduce ageing acceleration and therefore improve lifetime reproductive success. The enzymatic and non-enzymatic antioxidant scavengers, together with a variety of molecular repair processes, can help to limit immunopathology, and therefore ageing.

Given all these elements, we hypothesise that, in the context of ageing, fitness benefits from the storage of antioxidant carotenoids in crustaceans, especially astaxanthin for crustaceans, in dealing with inflammation consequences such as immunopathology, should be greater at early age than at older age. Given its biological properties mentioned earlier, this would be particularly the case for the storage of astaxanthin after its stabilisation as esterified forms for storage in crustacean tissues. ${ }^{[7,8,24]}$ This might also be particularly relevant in animals whose immunity relies almost exclusively on inflammatory responses, like again in crustaceans. After infection at early stages, carotenoids are expected to help keep the balance between pro- and anti-inflammatory status under control for a longer period of time, preventing ageing acceleration and preserving lifetime reproductive success (Fig. 3). Hence, maternal transfer of carotenoids into the eggs might be highly advantageous to prevent ageing acceleration in offspring in septic environments. ${ }^{[107,108]}$ In the particular case of crustaceans, we therefore predict that stored astaxanthin will have a greater positive effect on lifespan and early reproductive success early in life. In line with this, small gammarid crustaceans, that are the youngest, store higher quantities of carotenoids in their haemolymph than larger individuals, which are the oldest. ${ }^{[30]}$ Whether this age-related pattern of carotenoid storage is adaptive in the context of ageing is not yet known. Its consequences on lifetime reproductive success and ageing have to be tested. 

astaxanthin they accumulate. In addition of its high antioxidant activity, astaxanthin is able to

form chelate complexes with metal ions, such as $\mathrm{Zn}^{2+}$, which is known to inhibits the mitochondrial complex I (NADH: ubiquinone oxidoreductase) ${ }^{[109]}$, that is central for energy transduction and whose dysfunction is implicated in neurodegenerative and muscular diseases and in ageing. ${ }^{[29,73]}$

\section{Hypothesis 3: carotenoids storage is adaptive to fight pathogens}

Some organisms, including crustaceans, have evolved carotenoproteins (i.e. carotenoids in association with proteins $)^{[76,110]}$ that allow the storage of large amounts of carotenoids in their tissues for yet unknown reasons, and its involvement in sexual selection has never been reported so far (see the above section presenting crustaceans as relevant models systems to address the proposed hypotheses). Most crustaceans feed on decaying organic matter, probably full of obligate and opportunistic pathogens. ${ }^{[11]}$ Crustaceans also often live in microbes-rich aquatic environments that almost permanently expose their open circulatory system to microbial infections. ${ }^{[80-82]}$ From a host-pathogen interaction perspective, given the beneficial roles of carotenoids, especially astaxanthin, in the regulation of the immune function and associated immunopathology, ${ }^{[31,43,75]}$ we propose that, in these organisms, the storage of large amounts of carotenoids ${ }^{[30]}$ and the maternal transfer of carotenoids ${ }^{[7,28]}$ could have evolved to deal with pathogen attacks, using the more efficient inflammatory response provided by carotenoid enrichment. On the one hand, selection imposed by pathogens should favour resistance, giving rise to a more efficient immune response and higher levels of immune defences. ${ }^{[112,113]}$ On the other hand, since antioxidant defences are crucial to deal with the oxidative stress associated with the immune response and with the pathogens themselves, ${ }^{[114]}$ improved tolerance should be favoured simultaneously, yielding more 
efficient antioxidant defences through increased antioxidant enzyme production for instance. Hence, interactions with pathogens should increase broad sense immune costs, i.e. direct costs due to resource consumption by the immune response, and indirect costs resulting from immunopathology and the use of enzymatic antioxidant defences. ${ }^{[5,114-116]}$ The storage of immune-stimulant and antioxidant carotenoids should alleviate all these operating costs, providing crustaceans with a very efficient and lower-cost immune function. ${ }^{[30,31]}$ In this context, we hypothesise that there should be detectable correlations between the pathogen prevalence and the quantities of stored carotenoids among crustacean populations. Crustacean populations exhibiting higher pathogen prevalence, i.e. crustacean populations that are exposed to more frequent pathogen attacks, should evolve greater capacity of carotenoid storage in the tissues and/or in the haemolymph. While, on the one hand, such a relationship is likely driven by the potential benefits of storing large amounts of carotenoids under high pathogen pressure, on the other hand, it might also be driven by potential costs associated to carotenoids storage under low pathogen pressure.

Indeed, the above elements indicate that carotenoid storage is highly advantageous. However, natural variation in the amounts of stored carotenoids persist at each taxonomic level in animals, among phyla, ${ }^{[9]}$ species, ${ }^{[10]}$ and populations within species. ${ }^{[1]}$ This variation could reveal local differences in the environmental availability of carotenoids. It could also result from fitness costs associated with carotenoid storage. Under the hypothesis that carotenoid storage evolved to support the immune system in fighting pathogens, the expression of its costs should be maximal in the absence of pathogens. Moreover, the fact that carotenoid storage occurs despite bearing fitness costs would strongly support its adaptive nature. While detrimental effects of carotenoids have been suggested ${ }^{[12]}$ the potential fitness costs associated with their storage remain poorly investigated even in animals storing naturally these pigments. In copepod crustaceans, carotenoid reserves decrease the infection 
risk but increase the predation risk by making them conspicuous to predators. ${ }^{[117-119]}$ In birds, negative effects of high doses of carotenoids were reported on skeletal muscles and reproduction, mainly under relatively non-stressful conditions, suggesting context-dependent effects of carotenoids. ${ }^{[120,121]}$ Similarly, in the mealworm beetle, T. molitor, carotenoid supplementation provided larvae with a survival benefit under immune challenge, while it was associated with a survival reduction and prolonged larval development without immune challenge. ${ }^{[122]}$ Furthermore, at high concentrations, carotenoids may potentially reduce the immune response efficiency, through their high capacity to scavenge the free radicals released by the immune system, whose function is to kill pathogens. In some invertebrates, carotenoids may also potentially down-regulate the immune response by interfering with the cellular NO signalling, known as a major regulator of the insect immune response. ${ }^{[123-126]}$ Indeed, when NO stimulates both cellular and humoral immunity of insects, the strong antioxidant power of carotenoids may scavenge a fraction of circulating NO, thus down-regulating basal immune activity. In addition, certain carotenoids, such as astaxanthin, were reported to inhibit the enzymatic activity of the nitric oxide synthase responsible for NO production from L-arginin (Fig. 1), which activity is normally increased upon immune challenge. ${ }^{[19,124]}$ Such a general immune depressive effect of carotenoids was recently evidenced in $T$. molitor, increasing the susceptibility to entomopathogenic infections; ${ }^{[122]}$ It contrasts available evidence showing that carotenoids improve the immune response success in crustaceans. ${ }^{[30,31,43-45,89,90]}$ However, since crustaceans have evolved specialized features to store large amounts of carotenoids in their tissues in contrast to insects, ${ }^{[76]}$ these pigments might be of special importance in their physiology, including in immunity. This suggests that dietary carotenoids may be challenging for biological systems, at least for those that did not evolved specialized features to store them, and that beneficial and detrimental effects resulting from carotenoid diet supplementation might be host specific and context-dependent. Carotenoid storage might also 
have undiscovered costs, leading to spatial and temporal natural variation within and among invertebrate populations. It is therefore necessary to study the origins of natural variation in carotenoid content to know whether it is adaptive and to understand the conditions of carotenoid storage evolution.

Another important element for the storage of carotenoids to be adaptive is that its expression should be genetically or epigenetically encoded and therefore heritable so that natural selection can act on it. So far, no available studies have investigated whether the capacity to store carotenoids is heritable. Under the hypothesis that carotenoids storage is adaptive to fight pathogens, genetic variance and heritability for this trait might therefore be expected. Ample additive genetic variance and heritability have been found for components of the innate immune response in invertebrates. ${ }^{[127]}$ Investigating the genetic basis of carotenoid storage by measuring its heritability and estimating potential genetic correlations with components of the innate immune response as well as other life-history traits appears of primary importance. In particular, in addition to providing information on how much selection could act on the expression of carotenoid storage, this approach may also reveal to what extent it is associated to the expression of the innate immune response to pathogens.

\section{Conclusions and perspectives}

Despite its benefits against infections, the inflammatory immune response, through releasing oxidative cytotoxic molecules, has inevitable immunopathology costs, which decrease lifetime reproductive success and longevity. ${ }^{[32]}$ Endogenous antioxidant enzymes coupled with exogenous antioxidant compounds, including dietary carotenoids, help to limit immunopathology. ${ }^{[105]}$ Carotenoids are powerful antioxidants ${ }^{[35,36]}$ able to limit the oxidativemediated self-harm resulting from the immune response. Hence in addition to directly or indirectly stimulate immunity in some organisms ${ }^{[30,42-45,87,82,90]}$, carotenoids may limit the 
persistence of a pro-inflammatory status causing age-associated morbidity and death. ${ }^{[103,104]}$ Furthermore, carotenoids such as astaxanthin, may prevent metabolic wasting upon infection by influencing the host's metabolism regulation. ${ }^{[96,97,128]}$ Unlike other dietary antioxidants, carotenoids can be stored in animal tissues and, for yet unknown reasons, aquatic crustaceans exhibit particularly impressive capacities to store astaxanthin compared to other taxa. ${ }^{[7,9,66]}$ From these observations, we infer that greater carotenoid storage in crustaceans could be adaptive against pathogens.

To examine this possibility, we propose three testable hypotheses:

(1) As astaxanthin is antioxidant and immune-stimulant, the evolution of the crustacean immune function and enzymatic antioxidant defence system may have occurred in a particular context, because of their capacity to store large amounts of carotenoids. Large astaxanthin storage should therefore promote both resistance and tolerance to infection, providing crustaceans with a highly efficient immune response, but at low immunopathological costs. (2) As inflammation-associated immunopathology is closely linked to ageing, storage of carotenoids should prevent ageing acceleration resulting from early-life infection and therefore enhance lifetime reproductive success. In this context, maternal transfer of carotenoids into the eggs and the storage of these pigments early in life should be advantageous in a septic environment.

(3) By promoting resistance and tolerance, carotenoids may interfere in host-pathogen interactions, which would lead to correlations between these pigments storage and the pathogen prevalence among crustacean populations. Therefore, high pathogen pressure should favour higher capacity to store carotenoids.

These above roles of carotenoid storage might be particularly important in aquatic crustaceans, which live in microbes-rich environments constantly challenging their immune system, compare to other animals ${ }^{[80-82]}$, at least for two main potential reasons. First, the 
crustacean immune response strongly relies on hemocytes and their cytotoxic activity resulting from the prophenoloxidase system and ROS/RNS production, and marginally on the production of non self-reactive immune effectors such as the insect antimicrobial peptides and the vertebrate antibodies of the acquired immune system. ${ }^{[79]}$ Second, aquatic crustaceans have relatively large access to carotenoids in their habitat. ${ }^{[8-10]}$ One may speculate that such a large carotenoid availability may have prevented the evolution of immune innovations allowing a reduced use of ROS/RNS, like in insects.

\section{Author's contributions}

Y.M. conceived the ideas. A.B., J.M., and Y.M. performed the literature search and led the writing of the manuscript.

\section{Competing interests}

We have no competing interests.

\section{Funding}

This work was supported by the French National Research Agency (ANR- 08-JCJC-0006), the CNRS, and the Conseil Régional de Bourgogne.

\section{Acknowledgements}

We thank T. Rigaud for comments, and C. Chateau Smith for proof-reading our manuscript.

\section{References}

[1] G. A. Armstrong, J. E. Hearst, FASEB Journal 1996, 10, 228.

[2] N. A. Moran, T. Jarvik, Science 2010, 328, 624. 
[3] M. Grbic, T. Van Leeuwen, R. M. Clark, S. Rombauts, P. Rouzé, V. Grbic, E. J. Osborne, W. Dermauw, P. C. T. Ngoc, F. Ortego, P. Hernadez-Crespo, I. Diaz, M. Martinez, M. Navajas, E. Sucena, S. Magalhaes, L. Nagy, R. M. Pace, S. Djuranovic, G. Smagghe, M. Iga, O. Christiaens, J. A. Veenstra, J. Ewer, R. Mancilla Villalobos, J. L. Hutter, S. D. Hudson, M. Velez, S. V. Yi, J. Zeng, A. Pires-daSilva, F. Roch, M. Cazaux, M. Navarro, V. Zhurov, G. Acevedo, A. Bjelica, J. A. Fawcett, E. Bonnet, C. Martens, G. Baele, L. Wissler, A. Sanchez-Rodriguez, L. Tirry, C. Blais, K. Demeestere, S. R. Henz, T. R. Gregory, J. Mathieu, L. Verdon, L. Farinelli, J. Schmutz, E. Lindquist, R. Feyereisen, Y. Van de Peer, Nature 2011, 479, 487.

[4] A. Bendich, J. A Olson, FASEB Journal 1989, 3, 1927.

[5] A. P. Møller, C. Biard, J. D. Blount, D. C. Houston, P. Ninni, N. Saino, P. F. Surai, Avian Poult. Biol. Rev. 2000, 11, 137.

[6] P. F. Surai, B. K. Speake, N. A. R. Wood, J. D. Blount, G. R. Bortolotti, N. H. C. Sparks, Comp. Biochem. Phys. B 2001, 128, 743.

[7] M. Gaillard, C. Juillet, F. Cézilly, M.-J. Perrot-Minnot, Comp. Biochem. Phys. B 2007, 139, 129.

[8] T. Maoka, Mar. Drugs 2011, 9, 278.

[9] V. A. Olson, Oikos 2006, 112, 620.

[10] V. Ogilvy, A. L. Fidgett, R. F. Preziosi, Zoo Biol. 2012, 31, 470.

[11] S. Cornet, C. Biard, Y. Moret, Biol. Letters 2007, 3, 284.

[12] V. A. Olson, I. P. F. Owens, Trends Ecol. Evol. 1998, 13, 510.

[13] C. Isaksson, S. Andersson, Proc. R. Soc. London B, 2008, 275, 309.

[14] L. Pérez-Rodríguez, BioEssays, 2009, 31, 1116.

[15] P.A. Svensson, B.B.M. Wong, Behaviour 2011, 148, 131.

[16] M. J. P. Simons, A. A. Cohen, S. Verhulst, PLoS ONE, 2012, 7(8), e43088. 
[17] R. E. Koch, G. E. Hill, Funct. Ecol., 2018, 1.

[18] B. P. Chew, J. S. Park, J. Nutr. 2004, 134, 257S.

[19] G. Hussein, U. Sankawa, H. Goto, K. Matsumoto, H. Watanabe, J. Nat. Prod. 2006, 69, 443.

[20] J. S. Park, J. H. Chyun, Y. K. Kim, L. L. Line, B. P. Chez, Nutr. Metab. 2010, 7, 18.

[21] R. Castillo, G. Negre-Sadargeus, R. Lenel, General survey of the carotenoids in crustacea (Eds: G. Britton, T. W. Goodwin), Pergamon Press, Oxford, 1982.

[22] V. M. Dembitsky, T. Rezanka, Comp. Biochem. Phys. B 1996, 114, 383.

[23] F. Shahidi, J. A. Brown, Crit. Rev. Food Sci. Nutr., 1998, 38(1), 1.

[24] T. Matsuno, Fisheries Sci., 2001, 67, 771. [25] Blount \& McGraw 2008

[25] J. D. Blount, K. J. McGraw, in Carotenoids, Volume 4, Natural functions (Ed: G. Britton, S. Liaaen-Jensen, H. Pfander), Birkhäuser, Basel, 2008, pp 213-236.

[26] L. M. San-Jose, F. Granado-Lorencio, P. S. Fitze, Funct. Ecol., 2012, 26, 646.

[27] A. C. E. Rhodes, J. Plankton Res., 2007, 29, i73.

[28] R.J. Weaver, P.A. Cobine, G.E. Hill, J. Plankton Res., 2018, 40(2), 142.[29] L. A.

[29] Focsan, N. E. Polyakov, L. D. Kispert, Antioxidants, 2017, 6, 80.

[30] A. Babin, C. Biard, Y. Moret, Am. Nat. 2010, 176, 234.

[31] A. Babin, C. Saciat, M. Teixeira, J.-P. Troussard, S. Motreuil, J. Moreau, Y. Moret, Dev. Comp. Immunol. 2015, 49, 278.

[32] E. R. Pursall, J. Rolff, PloS ONE 2011, 6(5), e19972.

[33] G. W. Felton, C. B. Summers, Arch. Insect Biochem. 1995, 29, 187.

[34] C. Catoni, A. Peters, H. M. Schaefer, Anim. Behav. 2008, 76, 1107.

[35] A. El-Agamey, G. M. Lowe, D. J. McGarvey, A. Mortensen, D. M. Phillip, T. G. Truscott, A. J. Young, Arch. Biochem. Biophys. 2004, 430, 37. 
[36] S. Walrand, M.-C. Farges, O. Dehaese, N. Cardinault, R. Minet-Quinard, P. Grolier, C. Bouteloup-Demange, J. Ribalta, B. M. Winklhofer-Roob, E. Rock, M.-P. Vasson, Eur. J. Nutr. 2005, 44, 114.

[37] E. Sild, T. Sepp, M. Männiste, P. Hõrak, J. Exp. Biol., 2011, 214, 3467.

[38] R. E. Koch, A. N. Kavazis, D. Hasselquist, W. R. Hood, Y. Zhang, M. B. Toomey, G. E. Hill, Nature Com., 2018, 9, 491.

[39] J. H. Kim, Y. S. Kim, G. G. Song, J.-J. Park, H.-I. Chang, Eur. J. Pharmacol. 2005, 514, 53.

[40] D. H. Lee, C. S. Kim, Y. I. Lee, Food Chem. Toxicol. 2011, 49, 271.

[41] P. Schmid-Hempel, Evolutionary parasitology, Oxford University Press, Oxford, 2011.

[42] C. H. Pan, Y. H. Chien, J. H. Cheng, Zool. Stud. 2001, 40, 371.

[43] M. Flores, F. Diaz, R. Medina, D. Re, A. Licea, Aquac. Res. 2007, 38, 740.

[44] T. P. Sajeevan, D. W. Lowman, D. L. Williams, S. Selven, A. Anas, P. Rosamma, Aquac. Res. 2009, 40, 1723.

[45] B. Daly, J. S. Swingle, G. L. Eckert, Aquacult. Nutr. 2013, 19, 312.

[46] L. Cerenius, K. Söderhäll, Immunol. Rev. 2004, 198, 116.

[47] G. Sorci, B. Faivre, Philos. T. Roy. Soc. B 2009, 364, 71.

[48] A. J. Nappi, E. Ottaviani, BioEssays 2000, 22, 469.

[49] J. Weike, A. Wiesner, Nitric Oxide-Biol.Ch. 1999, 3, 123.

[50] A. J. Nappi, E. Vass, F. Frey, Y. Carton, Nitric Oxide-Biol. Ch. 2000, 4, 423.

[51] N. Hogg, V. M. Darley-Usmar, M. T. Wilson, S. Moncada, Biochem. J. 1992, 281, 419.

[52] A. Rivero, Trends Parasitol. 2006, 22, 219.

[53] L. Cerenius, B. L. Lee, K. Söderhäll, Trends Immunol. 2008, 29, 263.

[54] C.J. Coates, J. Nairn, Dev. Comp. Immunol., 2014, 45(1), 43. 
[55] B. M. Sadd, M. T. Siva-Jothy, Proc. R. Soc. B 2006, 273, 2571.

[56] I. Khan, D. Ahashe, J. Rolff, Proc. R. Soc. B 2017, 284, 20170125.

[57] S. J. Plaistow, L. Bollache, F. Cézilly, Anim. Behav. 2003, 65, 683.

[58] R. Stoks, F. Johansson, M. De Block, in Dragonflies and damselflies - Model organisms for ecological and evolutionary research (Ed: A. Cordoba-Aguilar), Oxford University Press, Oxford, 2008, pp. 39-51.

[59] M. T. Siva-Jothy, in Insect infection and immunity (Ed: J. Rolff, D. Reynolds), Oxford University Press, Oxford, 2009, pp. 241-250.

[60] J. Chaudière, R. Ferrari-Iliou, Food Chem. Toxicol. 1999, 37, 949.

[61] E. D. Harris, FASEB Journal 1992, 6, 2675.

[62] T. Holmblad, K. Söderhäll, Aquaculture 1999, 172, 111.

[63] M. W. Johansson, T. Holmblad, P. O. Thornqvist, M. Cammarata, N. Parrinello, K. Söderhäll, J. Cell Sci. 1999, 112, 917.

[64] D. D. Kitts, Trends Food Sci. Tech. 1997, 8, 198.

[65] R. K. Saini, S. H. Nile, S. W. Park, Food Res. Int. 2015, 76, 735.

[66] A. M. LaFountain, R. O. Prum, H. A. Frank, Arch. Biochem. Biophys. 2015, 572, 201.

[67] G.E. Hill, J.D. Johnson, Am. Nat., 2012, 180(5), e127.

[68] M.J.P. Simons, T.G.G. Groothuis, S. Verhulst, Ecol. Evol., 2015, 5(1), 224.

[69] D. Costantini, A. P. Møller, Funct. Ecol. 2008, 22, 367.

[70] P. Palozza, N. I. Krinsky, Arch. Biochem. Biophys., 1992, 297, 291.

[71] Y. M. A. Naguib, J. Agric. Food Chem., 2000, 48, 1150.

[72] M. Guerin, M. E. Huntley, M. Olaizola, Trends Biotechnol., 2003, 21, 210.

[73] N. E. Polyakov, A. L. Focsan, M. K. Bowman, L. D. Kispert, J. Phys. Chem. B, 2010, 114,16968 .

[74] M. A. Liñan-Cabello, J. Paniagua-Michel, P. M. Hopkins, Aquacult. Nutr. 2002, 8, 299. 
[75] I. Higuera-Ciapara, L. Felix-Valenzuela, F. M. Goycoolea, Crit. Rev. Food Sci. 2006, 46, 185.

[76] N. M. Wade, J. Gabaudan, B. D. Glencross, Rev. Aqua. 2017, 9, 141.

[77] J. Borgeraas, D. O. Hessen, Hydrobiologia 2002, 477, 15.

[78] Y.-H. Chien, C.-H. Pan, B. Hunter, Aquaculture 2003, 216, 177.

[79] L. Cerenius, P. Jiravanichpaisal, H.-P. Liu, I. Soderhall, in Invertebrate Immunity (Ed: K. Söderhäll), Springer, Boston, MA, 2010, pp 239-259.

[80] S. Nagasawa, Simidu, U., BioI., 1985, 87, 61.

[81] A. Niswati, M. Yamazaki, M. Ikenaga, M. Kimura, Soil Sci. Plant Nutr., 2002, 48, 185.

[82] K. G. Burnett, L. E. Burnett, Integr. Comp. Biol., 2015, 55(5), 856.

[83] L. Råberg, D. Sim, A. F. Read, Science 2007, 318, 812.

[84] J. S. Ayres, D. S. Schneider, Nat. Rev. Immunol. 2008, 8, 889.

[85] P. F. Vale, A. Fenton, S. P. Brown, PLOS Biology 2014, 12, e1001769.

[86] G. Sorci, Parasite Immunol. 2013, 35, 350.

[87] J. D. Blount, N. B. Metcalfe, T. R. Birkhead, P. F. Surai, Science 2003, 300, 125.

[88] R. J. Weaver, E. S. A. Santos, A. M. Tucker, A. E. Wilson, G. E. Hill, Nature Com., 2018, 9, 73 .

[89] V. Kumar, B. R. Pillai, P. K. Sahoo, J. Mohanty, S. Mohanty, Asian Fish. Sci. 2009, 22, 61.

[90] I. P. Jr Angeles, Y.-H. Chien, A. V. Yambot, J. Shellfish Res. 2009, $28,931$.

[91] C. Irna, I. Jaswir, R. Othman, D.N. Jimat, Int. Food Res. J., 2017, 24, S508.

[92] S. Weintraub, T. Shpigel, L.G. Harris, R. Schuster, E.C. Lewis, D.Y. Lewitus, Polym. Chem., 2017, 8, 4182 .

[93] Y. Yang, J. Min, A. Nguyen, T. X. Pham, H. J. Park, Y. Park, B. Kim, R. S. Bruno, J. Lee, J. Nutr. 2011, 141, 1611. 
[94] M. C. Powanda, W. R. Beisel, J. Nutr. 2003, 133, 322S.

[95] M. S. Dionne, L. N. Pham, M. Shirasu-Hiza, D. S. Schneider, Curr. Biol. 2006, 16, 1977.

[96] R. G. Fassett, J. S. Coombes, Mar. Drugs 2011, 9, 447.

[97] Y. Jia, J.-Y. Kim, H.-J. Jun, S.-J. Kim, J.-H. Lee, M.H. Hoang, S.-J. Um, H.I. Chang, S.J. Lee, Mol. Nutr. Food Res. 2012, 56, 878.

[98] M. R. Rose, Evolutionary Biology of Aging. Oxford University Press, Oxford, 2011.

[99] L. Kaszubowska, J. Physiol. Pharmacol. 2008, 59, 169.

[100] B. F. Oliveira, J. A. Nogueira-Machado, M. M. Chaves, The Scientific World J. 2010, 10,1121

[101] T. B. Kirkwood, S. N. Austad, Nature 2000, 408, 233.

[102] K. A. Hughes, M. Reynolds, Annu. Rev. Entomol. 2005, 50, 421.

[103] M. T. Lin, M. F. Beal, Nature 2006, 443, 787.

[104] F. Sesti, S. Liu, S. Q. Cai, Trends Cell Biol. 2010, $20,45$.

[105] C. E. Finch, E. M. Crimmins, Science 2004, 305, 1736.

[106] F. Licastro, G. Candore, D. Lio, E. Porcellini, G. Colonna-Romano, C. Franceschi, C. Caruso, Immun. Ageing 2005, $2,8$.

[107] A. C. Diaz, S. M. Velurtas, S. N. Mendiara, J. L. Fenucci, Invertebr. Reprod. Dev. 2013, 57, 43 .

[108] A. C. Brown, H. M. Leonard, K. J. McGraw, E. D. Clotfelter, Funct. Ecol. 2014, 28, 612.

[109] M. S. Sharpley, J. J. Hirst, Biol. Chem. 2006, 281, 34803.

[110] G. Britton, J. R. Helliwell, in Carotenoids, Volume 4, Natural functions (Ed: G. Britton, S. Liaaen-Jensen, H. Pfander). Birkhäuser, Basel, 2008, pp. 99-118.

[111] C. MacNeil, J. T. A Dick, R. W. Elwood, Biol. Rev. 1997, 72, 349. 
632

633

634

635

636

637

638

639

640

641

642

643

644

645

646

647

648

649

650

651

652

653

654

655

656

[112] K. M. Lindström, J. Foufopoulos, H. Pärn, M. Wikelski, Proc. R. Soc. B 2004, 27, 1513.

[113] M., Kalbe, J. Kurtz, Parasitology 2006, 132, 105.

[114] D. P. Parilla-Taylor, T. Zenteno-Savin, F. J. Magallon-Barajas, Aquaculture 2012, 41, 380.

[115] Y. Moret, P. Schmid-Hempel, Science 2000, 290, 1166.

[116] T. J. Little, S. C. Killick, J. Anim. Ecol. 2007, 76, 1202.

[117] I. T. Van der Veen, J. Evol. Biol. 2005, 18, 992.

[118] L.A. Hansson, Proc. R. Soc. B. : Biol. Sci., 2000, 267, 2327.

[119] E. Gorokhova, M. Lehtiniemi, N.H. Motwani, PLoS One, 2013, 8, e71385.

[120] K. A. Huggins, K. J. Navara, M. T. Mendonça, E. H. Geoffrey, Naturwissenschaften 2010, 97, 637.

[121] M. J. P. Simons, M. Briga, B. Leenknegt, S. Verhulst, Behav. Ecol. 2014, 25, 945.

[122] J. Dhinaut, A. Balourdet, M. Teixeira, M. Chogne, Y. Moret, Sci. Rep. 2017, 7, 12429.

[123] M. Imamura, J. Yang, M. Yamakawa, Insect Mol. Biol. 2002, 11, 257.

[124] A. R. Kraaijeveld, N. P. Elrayes, H. Schuppe, P. L. Newland, Dev. Comp. Immunol. 2011, 35, 857 .

[125] I. Eleftherianos, K. More, S. Spivack, E. Paulin, A. Khojandi, S. Shukla, Infect. Immun. 2014, 82, 4169.

[126] U. Sanzhaeva, Y. Vorontsova, Y. Glazachev, I. Slepneva, J. Enzyme Inhib. Med. Chem. 2016, 3, 1063.

[127] K. Hammerschmidt, P. Deines, A. J. Wilson, J. Rolff, Heredity 2012, 108, 569.

[128] G. Hussein, T. Nakagawa, H. Goto, Y. Shimada, K. Matsumoto, U. Sankawa, H. Watanabe, Life Sci. 2007, 80, 522. 
Figure 1. Diagrammatic representation of the respiratory burst during phagocytosis by

660

661

662

663

664

665

666

667

668 immune cells and the possible action of carotenoids (astaxanthin). Foreign objects are phagocytosed and encapsulated within a phagosome. NADPH oxidase is activated and accepts electrons from NADPH to reduce $\mathrm{O}_{2}$ to $\mathrm{O}_{2}{ }^{-}$( (superoxide anion). This anion could interact with nitric oxide (NO), produced by the inducible nitric oxide synthase (iNOS) from L-arginine to generate peroxynitrite $\left(\mathrm{ONOO}^{-}\right)$. Alternatively, $\mathrm{O}^{--}$could form hydrogen peroxide $\left(\mathrm{H}_{2} \mathrm{O}_{2}\right)$ by the superoxide dismutase (SOD) activity, which could be used by the catalase or other peroxidases (POD) in a process of detoxification to produce water $\left(\mathrm{H}_{2} \mathrm{O}\right)$ and oxygen $\left(\mathrm{O}_{2}\right)$. Alternatively, the myeloperoxidase (MPO) uses $\mathrm{H}_{2} \mathrm{O}_{2}$ in the presence of chloride $\left(\mathrm{Cl}^{-}\right)$, to generate hypochlorus acid $(\mathrm{HOCl}) . \mathrm{H}_{2} \mathrm{O}_{2}$ could also be used to produce again $\mathrm{O}_{2}{ }^{-}$ through Fenton's reaction or Haber-Weiss reaction. Carotenoids such as astaxanthin (ASTX; orange molecules) neutralize free radicals by scavenging singlet oxygen anions on both side of the cellular membrane. It could also inhibit the iNOS activity, thus limiting the production of NO. Explosive stars illustrate the cytotoxicity of the molecule inside.

Figure 2. Induction of the immune response (phagocytosis and prophenoloxidase cascade) after pathogen infection or wounding in crustaceans (plain grey arrows), and consequences on host homeostasis (plain and dashed arrows). The immune response can successfully clear infection by pathogens and repair wounds, leading back to homeostasis (plain grey arrow). Alternatively, pathogen clearance and wound healing may not be entirely successful, leading to increased self-harm, chronic inflammation and immunological oxidative stress (dashed arrows). 
Figure 3. Schematic view of the effects of the immune response on resistance to pathogens and immunopathology (black arrows) and the hypothetic action of dietary carotenoids in this system (orange arrows). Plus and minus signs refer to positive and negative effects on the arrow targets. The immune response helps to control the growth of pathogens entering the host, which is beneficial for host survival to pathogens. However, the immune response induces the production of ROS and RNS, which have negative impacts on host survival by inducing immunopathology and age-related diseases. Carotenoids might reduce the negative effects of ROS and RNS by scavenging free radicals, or by stimulating enzymes in the endogenous antioxidant system. Large filled orange arrows indicate the potential indirect consequence of increasing the strength of the immune response, leading to better resistance to pathogens. The large open dashed orange arrow with a plus sign between carotenoids and the immune response indicates the potential direct stimulatory power of carotenoids on the immune response. Thin dashed orange arrows indicate the carotenoid-mediated reduction of the negative effects of ROS and RNS that will also improve host survival by decreasing the probability of immunopathology and age-related diseases. 
Figure 1.

Intracellular

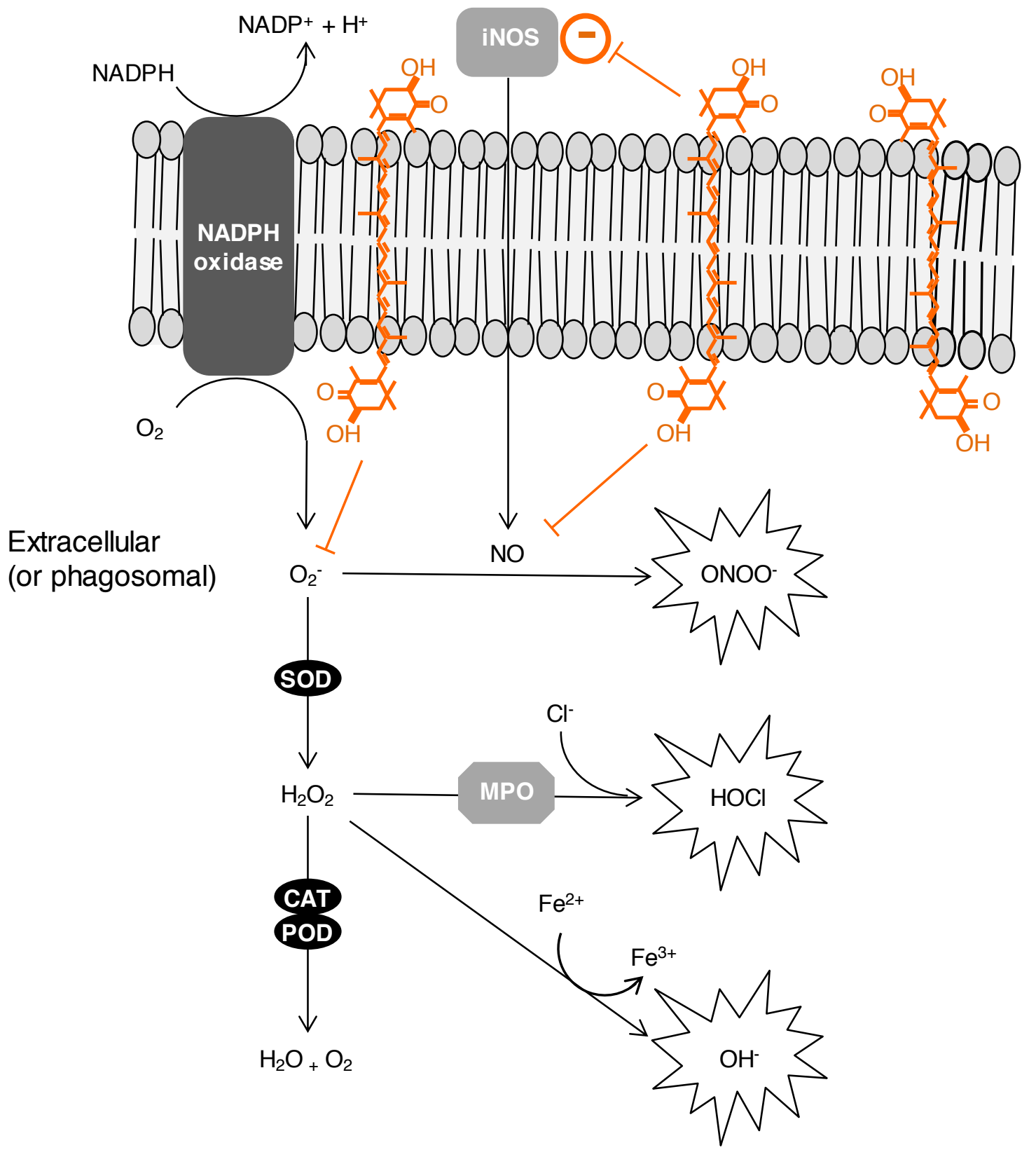


Figure 2.

Tissue/organ damages Chronic inflammatory response Immunological oxidative stress
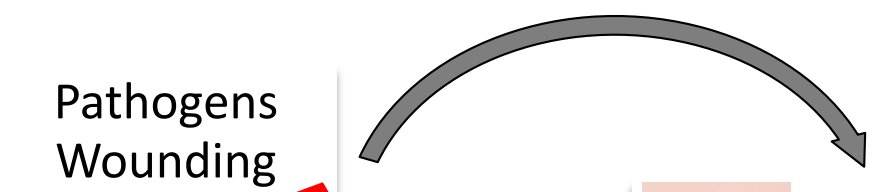

Phagocytosis ProPO cascade

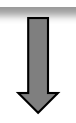

Melanisation

Cytotoxic products (ROS, RNS, quinone)

\section{Figure 3.}

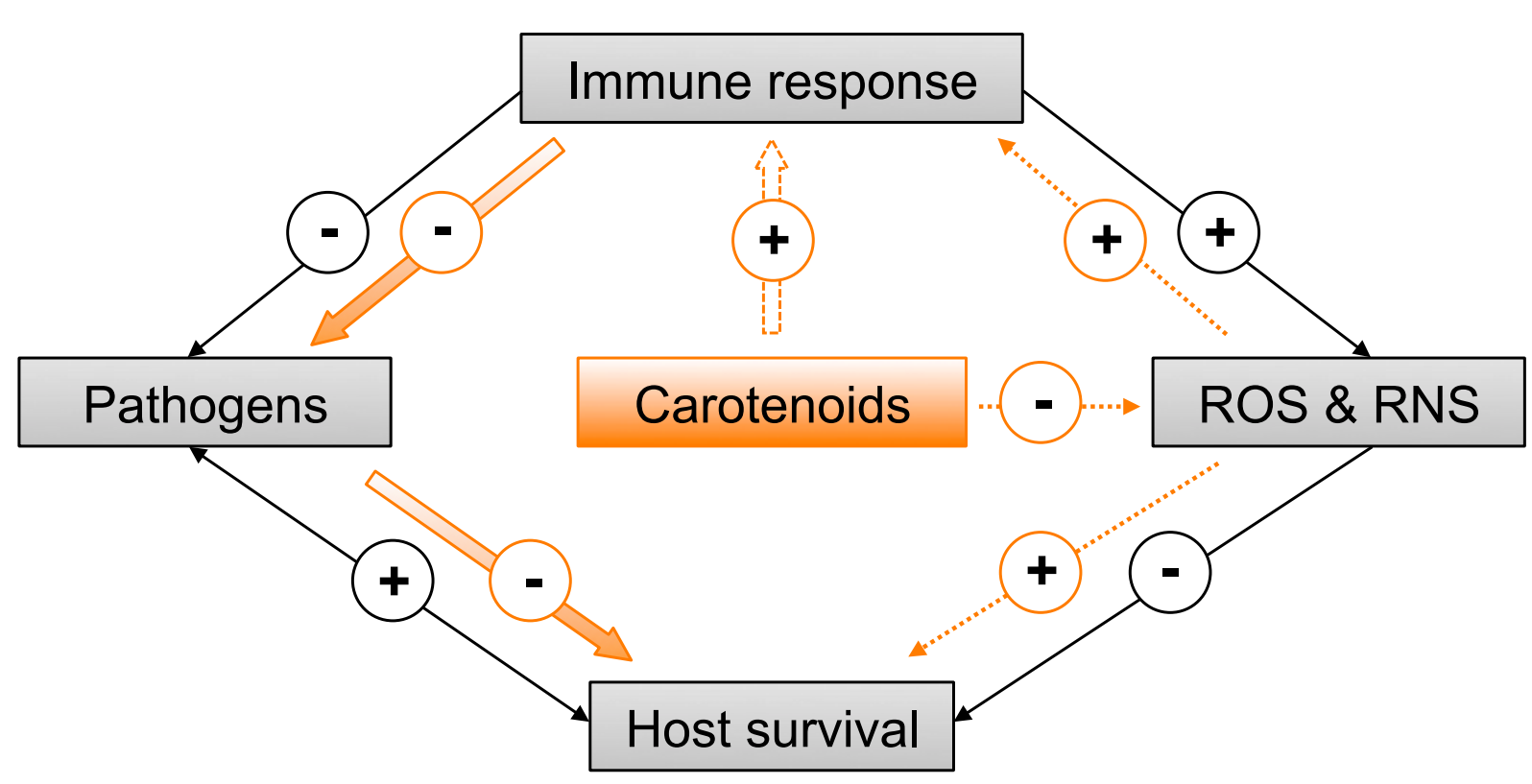

